\title{
Implementación de una Red Voip para una Empresa Textil
}

\author{
Implementing a VoIP Network for Textile Enterprise \\ Jorge Francisco Arimborgo Díaz
}

Universidad Alas Peruanas, N/A

\section{RESUMEN}

En los últimos años han surgido varias soluciones para Centros de Contacto para mejorar el nivel de servicio al cliente y abatir costos. Aunque estas soluciones están diseñadas para mitigar esta problemática, las redes convergentes de voz y datos basadas en Protocolo de Internet (IP) son la tecnología medular que facilita la funcionalidad mejorada, óptimo desempeño y aplicación eficaz de estas soluciones en el Centro de Contacto.

La tecnología de Voz sobre Protocolo de Internet (VolP) considerada compleja y cuestionable apenas hace unos cuantos años se está convirtiendo con rapidez en una tecnología convencional para el Centro de Contacto de hoy. . El nivel de conocimiento de la tecnología IP pura a nivel de usuario final en Centros de Contacto es sumamente bajo. A diferencia de otras soluciones para Centros de Contacto, como por ejemplo CRM multicanal, automatización del autoservicio y optimización de personal, la gran riqueza de beneficios y diferentes esquemas que existen para la tecnología IP no han sido transparentes para el mercado masivo

La tecnología de VoIP ha tenido una enorme evolución, desde aquella primera variante que permitía a los consumidores hacer llamadas gratuitas. En los últimos años, VolP se ha ramificado a partir de las aplicaciones orientadas al consumidor para incursionar en los terrenos empresariales. En la actualidad, la adopción y madurez de estándares tales como SIP y SS7, aunadas al surgimiento de una calidad de servicio (QoS) del orden de "cinco nueves" sobre redes IP (es decir, equiparable a la que ofrece la telefonía convencional), han abierto un nuevo abanico de oportunidades para las empresas en forma de Centros de Contacto IP. Y, hacia el futuro, es en el Centro de Contacto IP donde VoIP reportará los mayores beneficios estratégicos, pues ahí las empresas pueden liberar y aprovechar todo el potencial que encierra esta tecnología. 
Por eso al determinar la definición de nuestro tema llagamos a la conclusión que las deficientes percibidas en los procesos de comunicación que se dan a través de la VolP generar una deficiencia en los gastos que se generen en la empresa. Provocando de esta manera un error en la productividad y perdidas económicas de las mismas.

Descriptores: Protocolo de Internet (VoIP), estándares SIP y SS7

\section{ABSTRACT}

In recent years there have been several solutions for contact centers to improve customer service levels and reduce costs. Although these solutions are designed to mitigate this problem, the converged voice and data based on Internet Protocol (IP) core technology which provides enhanced functionality, optimum performance and effective implementation of these solutions in the contact center. The Voice over Internet Protocol (VoIP) considered complex and questionable just a few years ago is rapidly becoming a standard technology for the contact center today. . The level of knowledge of pure IP technology end-user level contact centers is extremely low. Unlike other contact center solutions such as multi-channel CRM, self-service automation and optimization of staff, the wealth of benefits and different schemes that exist for IP technology have not been transparent for the mass market VolP technology has had a tremendous evolution since the first version that allowed consumers to make free calls. In recent years, VolP has branched out from consumer-oriented applications to venture into the land business. Currently, adoption and maturity of standards such as SIP and SS7, coupled with the emergence of a Quality of Service (QoS) of the order of "five nines" on IP networks (ie, comparable to that offered by conventional telephony) have opened a new range of opportunities for companies in the form of IP contact centers. And the future is in IP contact center where the largest VolP bring strategic benefits because companies can then release and tap the full potential of this technology. So to determine the definition of our subject sores at the conclusion that perceived poor communication processes that occur through a deficiency in generating VolP expenses incurred in the company. Thus causing an error in the productivity and economic losses from them.

Descriptors: Internet Protocol (VoIP), SIP and SS7 standards

\section{INTRODUCCIÓN}

La comunicación es el medio por el cual se ejerce influencia sobre las personas para conseguir nuestros deseos o impartir órdenes, y por lo tanto es prioritario que la misma sea efectiva. El resultado de la comunicación esta dado por lo que al receptor entendió y no por lo que el emisor intento comunicar

También es muy importante la imagen usual de las personas, como sus expresiones y posturas, las cuales tiene una influencia muy superior a las palabras con las cuales no expresamos. Otro factor sobresaliente esta relacionado con los tonos de voz usados para comunicarnos.

La comunicación puede ser directa o indirecta, interpersonal o colectiva. Dentro de los procesos de comunicación se puede distinguir dos grupos: comunicación entre maquinas comunicación entre seres vivos, en esta ultima se observa la comunicación animal y la comunicación humana.
A su vez en la comunicación interpersonal se distinguen de acuerdo a la cantidad de personas, la comunicación interpersonal, la interpersonal, la intragrupal y la intergrupal. La comunicación dentro de las empresas es de gran importancia para que haya un buen desenvolvimiento de las relaciones humanas, y a su vez para que esta sea efectiva debe ser tratada con máxima seriedad, para lograr los objetivos que se quieran alcanzar.

La comunicación se clasifica en directa o indirecta, interpersonal o colectiva. Es directa cuando se efectúa sin intermediario de un emisor a un receptor, un dialogo, un conferencista a su auditorio. En la indirecta o se dan contactos inmediatos entre emisor $y$ recepto $y$, se interpone un intermediario.

Un documento, un escrito, una casete, un medio masivo. A partir de allí las posibilidades de comunicación se amplifican en el espacio y en el tiempo porque los medios se mantienen y 
multiplican. Dentro de la comunicación podríamos distinguir dos grandes grupos: la comunicación entre maquinas y la comunicación entre seres vivos, o social. A su vez, dentro de la comunicación observamos la comunicación animal y la comunicación humana.

La industria textil y de confecciones es uno de los sectores manufactureros de mayor importancia para el desarrollo de la economía nacional, por sus características y potencial constituye una industria integrada, generadora de empleo y que utiliza los recursos del pais.

En la actualidad la empresa Confecciones Rosateli's S.A.C esta especializada en la confección de ropa para caballero, damas y niñas, por ser una empresa pequeña, todos los pedidos se realizan según las especificaciones de los clientes, es decir, trabajan por órdenes específicas de producción. (descripción problemática)

Todas estas áreas deben interactuar, algunas de formas mas frecuente que otras dependiendo de la dimensión y el tipo de pedido solicitado para llevar a cabo el contacto con el cliente, producción del pedido, venta del producto, transporte del producto desde almacén hasta el cliente. Solo cuenta con cuatro áreas claramente definidas: administración, producción, almacén y ventas. Vienen laborando un total de 40 trabajadores.

La empresa actual mente cuenta con dos locales: EI primer local situado en Cooperativa Pachacutec Mz $\mathrm{H}$ lote 5 - Santa Anita consta de dos plantas donde se encuentran las oficinas de ventas $y$ administración, las cuales estarán ubicadas en el primer piso y segundo piso respectivamente.

- El segundo local situado en Cooperativa Pachacutec Mz E lote $9 \neg$ Santa Anita consta de tres niveles donde se encuentran el área de producción y almacén. En los dos primeros niveles se ubica el área de producción que cuenta en sus instalaciones con 18 maquinas de costura entre recta, remalle, recubierto, plana, dos maquinas bordadoras cada una con 8 cabezales y tres maquinas de corte. En el último nivel se ubica el almacén que se encuentra, separado del espacio dedicado a la venta, donde se guarda la mercancía.

Como cualquier otra empresa, la interacción entre los empleados tanto de una misma área como de distintas es indiscutible. El medio de comunicación mas utilizados por los empleados de los diferentes locales de una empresa, es la comunicación vía telefónica por ser la forma más cómoda rápida e interactiva.

Actualmente la empresa renta líneas telefónicas y también se encarga de administrar sus líneas utilizando una central privada conocida como PBX, la cual es proporcionada por el mismo proveedor de servicios.

La 1 PBX es un equipo de telefonía diseñado para lograr la conmutación de circuitos igual que en las centrales de conmutación pero con un menor numero de abonados como es una pequeña empresa. La red actual de la empresa a la cual se refiere la presente investigación cuenta con la siguiente infraestructura tecnológica para el proceso de comunicación de voz

Una central PBX Panasonic, can entrada para 3 líneas troncales analógicas ( 3 Líneas conectadas) y salida para 8 anexos de teléfonos análogos (8 teléfonos conectados) en el local principal distribuidos en las áreas de administración y ventas.

Además se cuenta con una línea telefónica analógica adicional en el local principal para el área de ventas. Dos Líneas telefónicas analógicas para el segundo local para las áreas de produccion y almacén respectivamente.

Asimismo, se cuenta con la siguiente infraestructura para la comunicaci6n de datos:

- Dos Líneas 3ADSL: una de 2048/512 Kbps y otra de 900/256 Kbps, 4switches y 5routers en el local principal distribuidos en las áreas de administraci6n y ventas.

- Una línea ADSL de 900/256 Kbps en el segundo local específicamente en el área de producci6n.

La comunicación entre el área de administración y el área de ventas se realiza a través de la central telefónica sin embargo la baja eficiencia que cumple la central PBX adquirida imposibilita la comunicaci6n sin costa entre el área de administración y ventas con el área de producción y almacén debido a que se encuentran en diferentes ubicaciones.

Al tener una regular afluencia de llamadas entre locales, que se encuentran ubicadas en diferentes 
zonas, el costo que se abona mensualmente por la comunicación entre empleados es un costo redundante ya que contando con una red de datos se puede aprovechar para transmitir voz y anular dicho costo del presupuesto mensual.

Cabe mencionar que se necesita una conexión de datos en el área de almacén para poder enviar información al área de ventas de la cantidad de productos que quedan en stock para dar mejor servicio en el menor tiempo posible al cliente.

El uso frecuente de la comunicación de voz entre las áreas de ventas y producción ocasiona un alto costo a la empresa a pesar de las rebajas de tarifas de la telefonea tradicional, los costos por usar una red dedicada exclusivamente a transmitir voz son mas altos debido a que se utiliza mayores recursos de hardware.

Necesidad de comunicarse con empleados que se encuentran fuera del local principal. Ya que la empresa tiene su área de producción en otro lugar y se requiere una comunicación constante entre locales esto genera un uso ineficiente de recursos económicos.

Debido a que se debe usar una línea telefónica (por 10 menú os) por cada local, con 10 cual se debe pagar además de renta fija los minutos utilizados durante la comunicación dejando la comunicación celular en algunos casos como la alternativa mas practica para realizar coordinaciones, sin embargo el costo de la telefonea celular es aun considerable, dependiendo incluso del lugar donde se encuentren

Si se tiene en cuenta el horario de operación de la empresa, de 9a. m. a 6p.m., podemos observar que no existe tarifa preferencial para estas llamadas al encontrarse dentro de los horarios donde se genera mayor cantidad de trafico.

No solo se produce mayores costos sino también menor productividad a la empresa debido alas Líneas telefónicas pertenecen a la central y otras son independientes al no contar con un orden en las Ígneas telef6nicas los empleados deben ubicar cual es el teléfono que tienen que contestar si es que no esta timbrando un teléfono perteneciente a su anexo.

El personal del área de administración son los encargados de contestar las Llamadas telefónicas lo cual provoca que dejen de lado sus labores para poder contestar las llamadas trayendo como consecuencia menor productividad en la empresa.
EXPERIMENTAL (METODOLOGÍA, SI EL TRABAJO ES NETAMENTE TEÓRICO)

Se describe el procedimiento experimental y el equipamiento utilizado. Aquí no se muestran los resultados sino se describe la metodología empleada en el trabajo experimental.

\subsubsection{Delimitación espacial}

El siguiente estudio de problema se lleva acabo en la cuidad de Lima, Perú en la empresa Confecciones Rosateli's S.A.C., con dirección Cooperativa Pachacutec Mz H lote 5 - Santa Anita

\subsubsection{Delimitación temporal}

El plazo requerido para la investigación es del 8 de Agosto del 2009 hasta el 30 de Noviembre del 2010

\subsubsection{Delimitación social}

Esta investigación se realizara en el área de comunicación de la empresa donde recibiremos la colaboración de la recepcionista y el técnico encargado de la central telefónica cuya función es la del mantenimiento de las redes de datos en las diversas áreas de la empresa.

\subsubsection{Delimitación conceptual}

Dentro de esta investigación cabe resaltar ciertos conceptos:

Central secundaria privada automática (PBX): es cualquier central telefónica conectada directamente a la red publica de teléfono por medio de líneas troncales para gestionar, además de las llamadas internas, las entrantes y/o salientes con autonomía sobre cualquier otra central telefónica

Este dispositivo actúa como una ramificación de la red primaria publica de teléfono, por 10 que 105 usuarios no se comunican al exterior mediante Líneas telefónicas convencionales, sino que al estar el PBX directamente conectado a la RTC (red telefónica publica), será esta misma la que enlute la llamada hasta su destino final mediante enlaces unificados de transporte de voz llamados Líneas troncales.

Línea troncal: es un enlace que interconecta las Llamadas externas de una central telefónica, concentrando y unificando varias comunicaciones simultaneas en una sola señal para un transporte $y$ transmisión a distancia mas eficiente (general mente digital) y poder establecer comunicaciones con otra central 0 una red entera de ellas. 
ECIPERÚ

ADSL: Línea de Suscripción Digital Asimétrica. Consiste en una transmisión de datos digitales (la transmisi6n es anal6gica) apoyada en el par simétrico de cobre que leva la línea telefónica convencional o línea de abonado, siempre y cuando el alcance no supere $1055,5 \mathrm{~km}$. medidos desde la Central Telefónica, 0 no haya otros servicios por el mismo cable que puedan interferir.

Smith: Conmutador. Es un dispositivo digital de lógica de interconexi6n de redes de computadores que opera en la capa 2 (nivel de enlace de datos) del modelo OSI. Su función es interconectar dos 0 mas segmentos de red, pasando datos de un segmento a otro de acuerdo con la dirección MAC de destino de las tramas en la red.

Router: Enrutador. Es un dispositivo de hardware para interconexión de red de ordenadores que opera en la capa tres (nivel de red). Un router es un dispositivo para la interconexión de redes informáticas que permite asegurar el enrutamiento de paquetes entre redes o determinar la ruta que debe tener el paquete de datos

\subsection{Objetivos de la investigación}

\subsubsection{Objetivo general o principal}

Determinar cuales son las limitaciones que ofrece una red de VolP dentro de la empresa de Confecciones Rosateli's S.A.C., y que consecuencia conlleva a disminuir los recursos de la misma empresa

1.5. Justificación, importancia y limitaciones del estudio

\subsubsection{Justificación}

Una creencia errónea muy extendida acerca de los Centros de Contacto IP puros es su supuesta falta de confiabilidad. La respuesta es no. En los últimos dos años, los proveedores de servicios de comunicaciones de datos por conmutación de paquetes han logrado consolidar una infraestructura robusta y redundante que hoy por hoy ofrece un alto nivel de confiabilidad del servicio. Asimismo, la utilización del esquema IP puro sobre la red de datos empresarial permite a los Centros de Contacto tener una mayor autonomía sobre el monitoreo del desempeño y la localización de fallas.

Los sistemas IP más modernos, como por ejemplo Nazis NuContact Center, cuentan con nueva funcionalidad para garantizar la confiabilidad $y$ disponibilidad de la red datos. Por ejemplo, como parte de NuContact Center, Nuasis ofrece una herramienta que detecta fallas en los servidores y redes locales y automáticamente canaliza las llamadas entrantes a otros Centros de Contacto.

Los proveedores de aplicaciones de administración también han creado herramientas para detectar con gran precisión dónde se encuentra una falla en una red IP pura, de modo que los Centros de Contacto puedan desviar las llamadas y mitigar el impacto de un cuello de botella o falla.

\subsubsection{Importancia}

Tradicionalmente, la industria de la telefonía trabaja con unas altas exigencias de fiabilidad, conocidas como los "cinco nueves": 99,999 por ciento. Esto se traduce en unos objetivos de diseño de centrales públicas de conmutación que garantizan niveles de caída del servicio de sólo dos horas cada cuarenta años de operación.

El crecimiento y fuerte implantación de las redes IP, tanto en local como en remoto, el desarrollo de técnicas avanzadas de digitalización de voz, mecanismos de control y priorización de tráfico, protocolos de transmisión en tiempo real, así como el estudio de nuevos estándares que permitan la calidad de servicio en redes IP, han creado un entorno donde es posible transmitir telefonía sobre IP lo que no significará en modo alguno la desaparición de las redes telefónicas modo circuito, sino que habrá, al menos temporalmente, una fase de coexistencia entre ambas, y por supuesto la necesaria interconexión mediante pasarelas, denominadas genéricamente pasarelas VoIP.

\subsubsection{Limitaciones}

Frente al constante cambio de las telecomunicaciones, la telefonía sobre IP es excepcionalmente prometedora. Ante un mercado global cada vez más competitivo, las compañías telefónicas ya existentes, los proveedores de servicios de Internet, las operadoras locales competitivas emergentes buscan, en forma constante, maneras de aumentar sus ofertas de servicios.

La telefonía sobre IP ha captado la atención de dichos proveedores de servicios en todo el mundo, ofreciendo una amplia gama de servicios nuevos $y$ reduciendo al mismo tiempo sus costos de infraestructura. La voz sobre IP (Voice over IP VoIP) 
está cambiando el paradigma de acceso a la información, fusionando voz, datos, facsímile $y$ funciones multimedia en una sola infraestructura de acceso convergente.

Mediante la telefonía sobre IP, los proveedores de servicios pueden ofrecer servicios de voz básicos $y$ ampliados a través de Internes, incluyendo la llamada en espera en Internet, el comercio en la web por telefonía ampliada y comunicaciones interactivas de multimedia. Estos servicios se integrarán de manera ininterrumpida a las redes conmutadas existentes a fin de permitir que se originen 0 terminen llamadas en teléfonos tradicionales según sea necesario. Dado que IP es una norma abierta, VolP le brinda a los proveedores de servicios flexibilidad para personalizar sus servicios existentes e implementar nuevos servicios con mayor rapidez $y$ eficiencia en función de los costos que antes, incluso en áreas remotas dentro de su región

La voz sobre IP convierte las señales de voz estándar en paquetes de datos comprimidos que son transportados a través de redes de datos en lugar de líneas telefónicas tradicionales. En la actualidad, se están proponiendo otras especificaciones en los consorcios industriales tales como SIP, SGCP e $I P D C$, las cuales ofrecen ampliaciones en lo que respecta al control de llamadas y señalización dentro de arquitecturas de voz sobre IP.

Tabla:

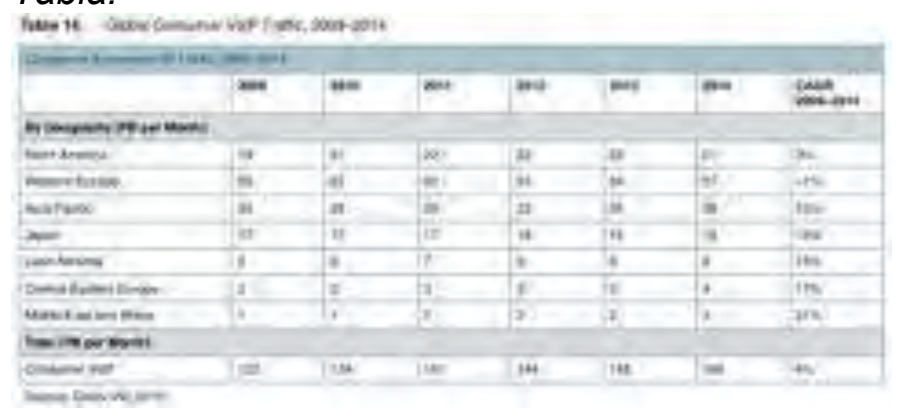

\section{RESULTADOS Y DISCUSIÓN}

En resumen se logro una aplicación basada totalmente en herramientas libres cumpliendo con el licenciamiento GPL, que cubre el ambiente educativo y técnico-profesional, teniendo como resultado un software capaz de dar respuesta con basamentos robustos al momento de una implementación de VolP en ambientes laborales pero de igual forma puede ser usada como herramienta didáctica a distintos niveles de enseñanza del área relacionada a VolP.

1. Aumentar los modelos de codecs a escoger como parámetro.

2. Flexibilizar de los parámetros de selección.

3. Incluir modelos de simulación.
4.Poner a disposición del área estudiantil y profesorado de las distintas casas de estudios superiores que imparten conocimientos relacionados al mundo de las telecomunicaciones y VoIP. 23

\section{CONCLUSIONES}

Se desarrollaron satisfactoriamente cada una de las fases necesarias para culminarla. Se desarrolló un diagnóstico de la problemática planteada a través de los diferentes recursos utilizados (humanos, bibliográficos, tecnológicos), de su ámbito, usuarios y necesidades. Luego de todo el análisis, se realizó el diseño y posterior desarrollo del software de la herramienta para finalmente evaluar su funcionalidad.

La herramienta muestra a los usuarios los parámetros establecidos por la ITUT, y en forma flexible le permite configurar los parámetros de suma importancia como son: jitter, delay y perdida de paquetes típico según la función de los elementos de red para generar los cuales permiten realizar los cálculos que influyen en la obtención del resultado.

La herramienta emite de manera informativa, observaciones y recomendaciones que el usuario necesita conocer para el logro satisfactorio de su análisis.

- Se logró el desarrollo de una herramienta que permite a un consultor-especialista, educador o estudiante obtener resultados debidamente soportados para la implementación o el estudio de la VolP.

\section{AGRADECIMIENTOS}

Agradezco a mis compañeros de la Universidad por brindarme todo su apoyo en este proyecto.

\section{REFERENCIAS}

[1] Ing. José M. Figueredo A. Herramienta de Evaluación para la Implementación Eficaz de Voip en las Organizaciones bajo Software Libre. Barquisimeto. Junio 2011.

[2]James F. Ransome, John W. Rittinghouse. Voip Security. Elsevier Digital Press. Año 2005

[3] J. Mallery, J. Zann, P. Kelly, W. Noonan, E. Seagren, P. Love, R. Kraft y M. O'Neill. Blindaje de Redes. Tu red invulnerable a los hackers. Anaya Multimedia

[4] Michal Zalewski. Silence on the Wire A Field Guide to Passive Reconnaissance and Indirect Attacks. No Starch Press. Año 2005

[5] Richard Bejtlich. El Tao de la Monitorización de Seguridad de Redes. Pearson-Addison Wesley. Año 2005

E-mail: diazj231@hotmail.com 